\title{
DSM AND DTM FOR EXTRACTING 3D BUILDING MODELS: ADVANTAGES AND LIMITATIONS
}

\author{
F. Fissore ${ }^{1,2}$, F. Pirotti ${ }^{1,2 *}$ \\ ${ }^{1}$ CIRGEO - Interdepartmental Research Center of Geomatics, University of Padova, Viale dell’Università 16, Legnaro \\ (PD), Italy - francesca.fissore@unipd.it, francesco.pirotti@unipd.it \\ 2 TESAF Department, University of Padova, Viale dell’Università 16, Legnaro (PD), Italy
}

Commission IV, WG IV/4

KEY WORDS: CityGML, Digital terrain model, Digital surface model, Lidar

\begin{abstract}
:
Using multiple sources of 3D information over buildings to go from building footprints (LOD0) to higher LODs in CityGML models is a widely investigated topic. In this investigation we propose to use a very common 2.5D product, i.e. digital terrain and surface models (DTMs and DSMs), to test how much they can contribute to improve a CityGML model. The minimal information required to represents a 3 dimensional space in an urban environment is the combination of a DTM, the footprints of buildings and their heights; in this way a representation of urban environment to define LOD1 CityGML is guaranteed. In this paper we discuss the following research questions: can DTMs and DSMs provide significant information for modelling buildings at higher LODs? What characteristics can be extracted depending on the ground sampling distance (GSD) of the DTM/DSM? Results show that the used DTM/DSM at $1 \mathrm{~m}$ GSD provides potential significant information for higher LODs and that the conversion of the unstructured point cloud to a regular grid helps in defining single buildings using connected component analysis. Regularization of the original point cloud does loose accuracy of the source information due to smoothing or interpolation, but has the advantage of providing a predictable distance between points, thus allowing to join points belonging to the same building and provide initial primitives for further modelling.
\end{abstract}

\section{INTRODUCTION}

Urban areas are continuously changing due to construction and extension of buildings. Dynamic and accurate update of geometries in a cartographic context is very important for multiple reasons, ranging from cadastre to land-cover analysis and $3 \mathrm{D}$ analysis for city management. Three-dimensional urban models are increasingly needed for applications as varied as urban planning and design, energy studies and tourism (Morgan and Habib, 2002; Steed et al., 2004).

Representing geographical information of urban environment in a 3D space is becoming a common reality thanks to advancing technology in computer vision. This has found large interest in the scientific community engaged in numerous fields related to urban and natural environments because it can be used to simulate and apply models that can come closely represent the real world (Agugiaro, 2016). Virtual world representation are becoming current also on virtual globes, Google Earth being the most known, but also open source implementations like NASA's World Wind (Pirotti et al., 2017).

Currently, there are several types of 3D standards and tools for displaying 3D geospatial data sets. Between standards, OGC CityGML is one of those that allow to represent together geometries and attributes. Moreover, the CityGML model offers the possibility to exchange and save easily the 3D dataset, since it has been developed as open standard model based on the xml format. CityGML is also extremely extensible through its application domain extensions (ADEs) - as described in
(Agugiaro et al., 2018; Biljecki et al., 2018; Labetski et al., 2018; M. et al., 2000).

The minimal information required to represents a 3 dimensional space in an urban environment is the terrain model (DTM), the footprint of buildings and their height; in this way the representation of urban environment to the level of detail LOD1 is guaranteed. Other information related to the urban environment (e.g. roof type, number of floors, type of buildings) can be used as additional information to enrich semantics of the 3D model. There are tools available that provide the capability of automatically creating LOD1 CityGML from this information. TUDelft's 3dfier (Arroyo Ohori et al., 2018; TUDelft, 2019) finds building heights from LiDAR data in LAS/LAZ format and converts them to CityGML objects. Also roads and rivers are inserted in the 3D output, with topologically coherent objects (no sliver or gapped polygons). The software suite Feature Manipulation Engine - FME - was tested in an investigation (Sengul, 2012) and provides steps to extrude polygons with other data. These and further investigations are important because there are many datasets available from past surveys that are not used as 3D data, but do contain 3D information. For example digital cartography - geotopographic databases - LiDAR data, photogrammetric products etc... The biggest obstacle to fully exploit this information is variety, complexity and the heterogeneity of the source data formats.

In recent years, the use of LiDAR data to model the urban environment has received greater attention due to the speed to collect $3 \mathrm{D}$ information about an urban site and the integration of 
data with GNSS (Palmer and Shan, 2002; Verma et al., 2006). LiDAR data are very suitable for 3D reconstruction of urban features and have been widely investigated (Alharthy and Bethel, 2001; Zeng, 2008). Application to CityGML modelling comes as a natural consequence and has been investigated through many approaches. In (Jayaraj and Ramiya, 2018) the details of the roof model is extracted using ArcGIS Pro tools. The tool tries to fit standard roof models to the LiDAR points that are labelled as roofs, and then fixes slope and aspect by analysing the residuals between the surfaces that are the output of the modelling and the points.

In the detection and estimation of 3D elements of buildings from unstructured point clouds, one of the main issues is linked to the identification of the edges. This difficulty can be solved using additional information (commonly 2D information) concerning the shape of the foot of the buildings that are retrieved through cartographic data. Detection of objects in point clouds is a widely investigated topic, with supervised classification of contextual features and segment-based features has given positive results (Vosselman, 2013; Weinmann et al., 2015) also regarding roof and façade detection (Barazzetti et al., 2010; Pirotti et al., 2019).

Within the Urban-Geo Big Data project (Brovelli et al., 2017) an Italian project of national interest (PRIN 2015), a large amount of cartographic data related to some of the main Italian cities was collected. Amongst the targets of the project, there is a need to identify standards for the extract transform and load (ETL) process of conversion from cartographic vector models to $3 \mathrm{D}$ CityGML models.

There are many solutions that convert in a semi-automatic way cartographic data to CityGML model. A custom solution used here, called shp2city (Fissore and Pirotti, 2018), was developed in Python, and uses as input the polygons of building footprints and uses attribute data from the input polygon or data from other vector files that have information on building height. In the latter case, a spatial join allows to associate the building footprint with the other data source. The output of shp2city is a 3D representation of the urban environment at LOD1, where footprints, the height of buildings and all the attributes used to enrich the 3D model are obtained by processing information present in the input geospatial data. The first version of shp2city was developed to processes only geospatial vector data format to generate CityGML model, simply extracting footprint from the geometry and building height from attributes. The success of creating the $3 \mathrm{D}$ model is of course limited to the availability of correct building height information in the attribute tables.

Therefore, in the second version we are interested the possibility to obtain information of buildings height using indirect ways such as using LiDAR-derived models and cartography with machine learning models. (Alharthy and Bethel, 2001; Rottensteiner, 2008; Rottensteiner and Briese, 2002). In this solution information from lidar-derived terrain and surface models is used. This paper will address the problem of being able to integrate the cartographic data with information obtained from LiDAR-derived data DTMs and DSMs in order to: i) validate the information included in the cartographic dataset, ii) integrate missing data (mostly building height and roof type) (Lu et al., 2014; Verma et al., 2006) iii) establish a proof of concept for creation of a 3D cityGML model with accurate and rich of information. The results showed in this paper are related to the city of Naples of which we have access to cartographic data at 1:10000 scale, with height of each building, and DTM and DSM datasets obtained with LiDAR technology.

\section{STUDY AREA AND DATA}

\subsection{Study Area}

The metropolitan city of Naples with a population of over three million people is the third metropolitan Italian city by number of inhabitants, while it is first in population density. The entire metropolitan area covers an area of $1,171 \mathrm{~km}^{2}$ and includes 92 municipalities.
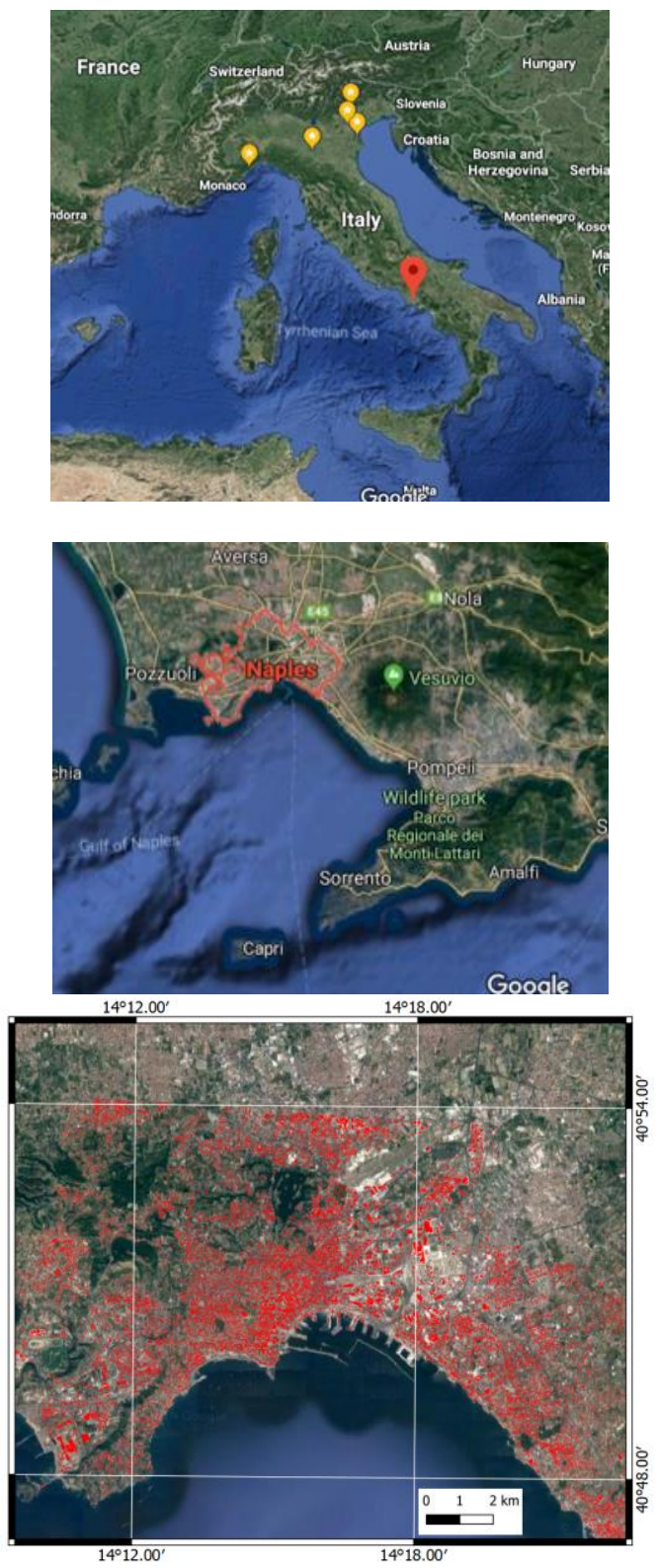

Figure 1. Metropolitan City of Naples (Google Maps 2019)

The main feature that distinguishes the city of Naples from other Italian metropolitan cities are: i) its seismic and volcanic vulnerability, due to the presence of Vesuvius and the Phlegraean 
Fields, ii) high concentration of the population on a restricted geographical area (on an area that cover just $8.6 \%$ of the area of Campania region, more than half of the entire regional population is concentrated). This phenomenon of overcrowding has created a strong demographic and territorial imbalance with the other areas of the region, Naples is nevertheless Italy's 3rd largest city by population, making it one of the most densely populated areas in Europe; the metropolitan region also includes the municipality of Casavatore, the highest-density municipality in Italy (at 12,000 inhabitants $/ \mathrm{km}^{2}$ ).

\subsection{Datasets}

Related to Naples' city, we have available, as open data, cartographic data and LiDAR data. The data were obtained by the national cartographic portal (Ministero dell'Ambiente e della Tutela del Territorio e del Mare, 2019).

1.2.1 Accuracy of data: the objective of this investigation is to understand to what extent can LiDAR-derived products be used to model building heights and roof details. To achieve this cartographic data at 1:10000 scale and LiDAR DTM and DSM datasets with $1 \mathrm{~m}$ ground sampling distance (GSD) were used. The cartographic data in ESRI Shapefile format contained building footprints and attributes regarding height above sea level of building foot and heaves. Digital models were obtained with LiDAR aerial survey surveyed in December 2009, with a point density of $\sim 4$ points $/ \mathrm{m}^{2}$, and accuracy of $\sim 15 \mathrm{~cm}(1 \sigma)$. This allowed to create a dense (level 6) digital terrain/surface model of, i.e. with vertical and horizontal expected accuracy of $0.3 \mathrm{~m}$ (Brovelli et al., 2012)

The building footprints and heave heights were available as shapefile data at 1:10000 scale. This means that geometric vertical accuracy is expected to be $2 \mathrm{~m}(1 \sigma)$, thus related to a level 2 DSM accuracy (Brovelli et al., 2012). Comparing this with the DTM and DSM expected accuracy shows that the most accurate product is expected to be the digital terrain models.

It might seem like a trivial task, but variety and accessibility of the data, which is different for each of the five cities considered, increase complexity. It is worth noting that geographic data harmonization is among the goals of regional and national (and European) policies; there is still a lot of archived data that contain information that can enrich urban models; therefore, ETL will continue to be a very important aspect for this topic. In the methodology we will present only the city of Naples.

\section{METODOLOGY}

In order to extract information of buildings height, we used the 1 meter resolution LiDAR derived Digital Surface Model (DSM) and Digital Terrain Model (DTM) from which we calculate normalized DSM (nDSM) as the difference between DSM and DTM. The initial step is to extract a raster with difference between DSM and DTM to have a normalized height map that theoretically has a value of zero over bare ground and roof height over buildings. This is then converted to a regularly spaced point cloud representing the center of the cell. We therefore obtain a distribution of values of height every $1 \mathrm{~m}^{2}$.
Intersecting each point grid with the polygons described in the cartographic dataset, that represent the footprint of buildings, we obtain both a distribution of height values at heaves for each building - using the lowest points inside the polygon - and the distribution of heights that represent the roof shape. These values can be used to (i) obtain values of building elevation in the cartographic dataset (ii) carry out statistical surveys aimed at estimating the type of roof (main objective of this work), and (iii) analyse residuals and discuss applicability of assigning building height from lidar-derived raster height models.

Because we are going to compare height of buildings measured at the heaves, we assign LiDAR-derived height of a building as the median of the lowest 20 points in each building footprint. The use of the median allows to have a value that is more robust in case of outliers than using the mean. Outliers can consist in points belonging to wall reflections or higher roof parts.

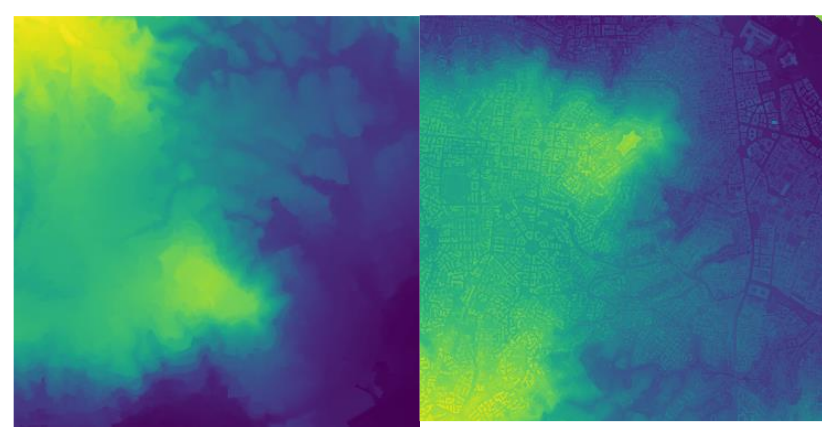

(a)

(b)

Figure 2. The LiDAR DTM (a) and DSM (b) of Naples

\section{RESULTS AND DISCUSSION}

A total of $\sim 11$ million points, i.e. cell centers, overlap building footprints. Residuals between from lidar-derived raster height models and heights of buildings from cartographic shapefiles attributes are shown in Figure 4 below. It was calculated by finding the lowest roof-point in each polygon representing the building footprint, and subtracting it from the building absolute height at heave, derived from adding building height at heave to $\mathrm{Z}$ value of polygon to get height above sea level. LiDAR heights are referred to geoid height above mean sea level. Results show differences in all buildings analysed (15000 buildings). Distribution of residuals between cartographic building heights and LiDAR-derived heights have an average of $-1.3 \mathrm{~m}$ and a standard deviation of $4.15 \mathrm{~m}$. This result is in line with accuracy that is expected from the 1:10000 scale, considering higher residuals due to errors defining the lidar point in the roof that represents heave heights. 


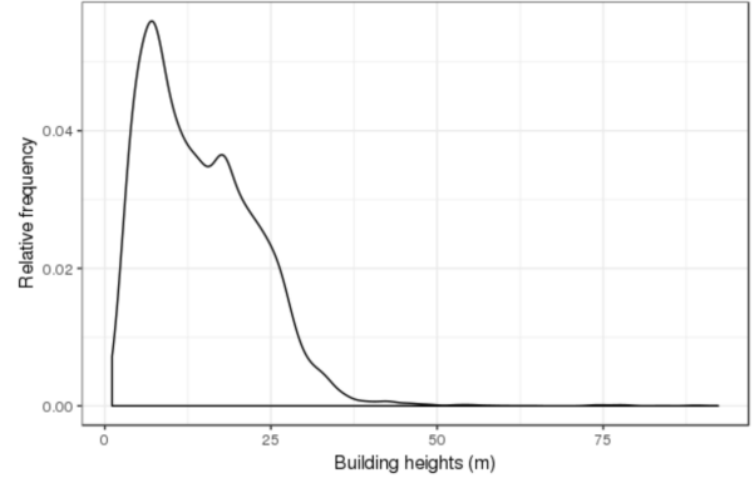

Figure 3. frequency of building heights as recorded in cartographic sources

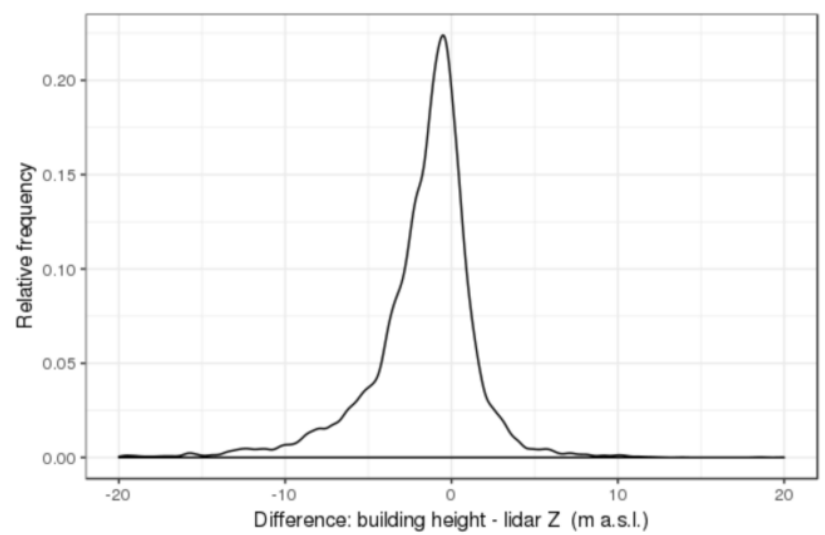

Figure 4. Residuals frequency distribution calculated by adding building height to ground height above sea level and subtracting lidar height

Most points are on roofs, but some also are from facades and walls, as the LiDAR point density used was high enough to also reflect significantly from walls as is shown in Figure 5.

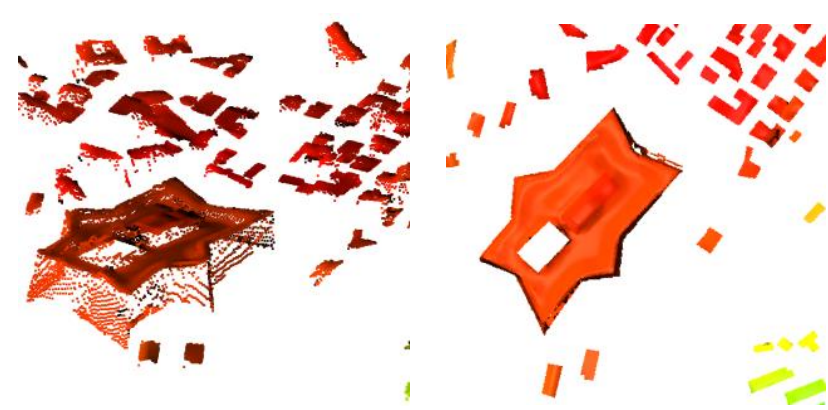

Figure 5. Castel San Elmo Naples with detail in the left of points on the wall.

The planar projection of points will have a $1 \mathrm{~m}$ step, but the 3D representation will increase the reciprocal distance depending on the slope of the surface that was sampled by the grid's cell center. Spurious points are also visible as the fall in the building footprint, but do not belong to the roof or façade. This can be cleaned out by one of the many outlier filters that are available such as discussed in Pirotti et al., (2018).

\subsection{Extraction of single buildings with connected components}

Connected component analysis is used in this work to try and isolate single buildings. The connected component labelling algorithm was implemented in the computer vision community to detect connected elements in binary digital images (Dillencourt et al., 2002).

Application over unstructured point clouds has been investigated diffusely to support segmentation methods and object detection. Many methods have been implemented in various software. We have used CloudCompare and applied its version of connected components labelling. The tool provides two parameters for tuning, the octree level and the minimum number of points per segment. Higher octree levels tend to under-connect, thus splitting point clusters belonging to the same building, whereas lower octree levels tend to over-connect, thus grouping large chunks of points also from multiple buildings. Results are shown in Figure 6 below.
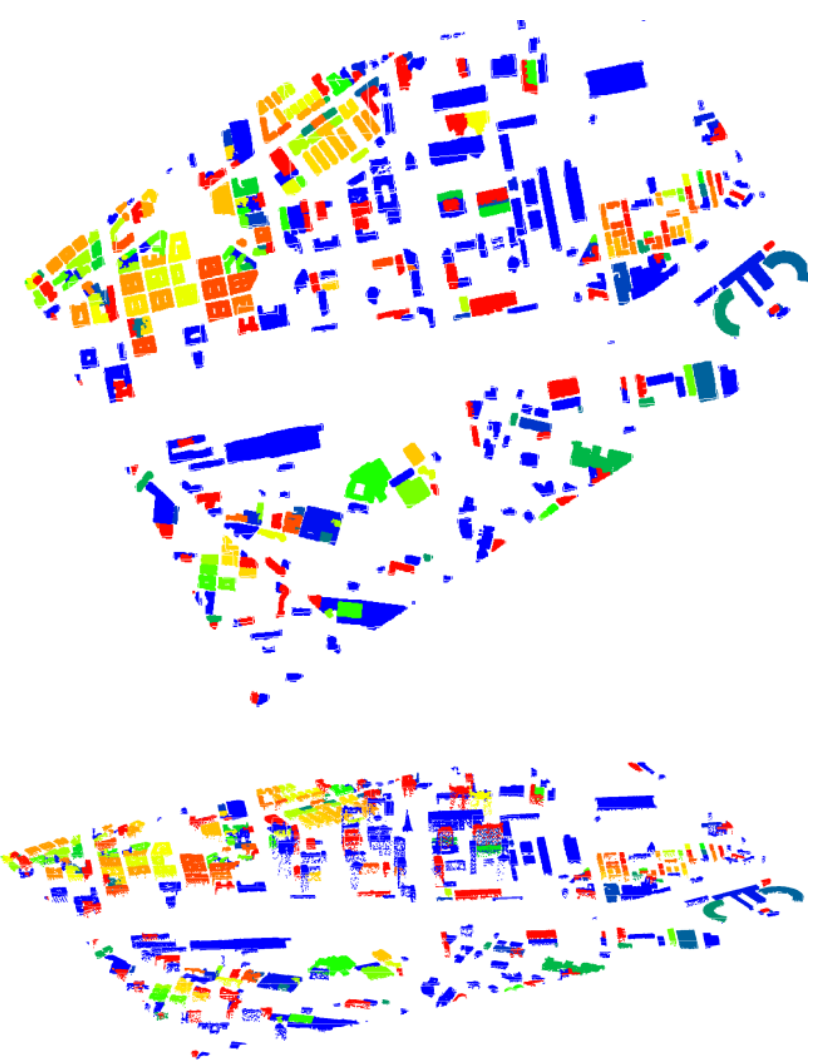

Figure 6. Top and perspective view of connected components results.

A triangulation process over points, with a maximum edge length filter, can isolate building roofs partially as shown in Figure 7 below. The regularly spaced points help in defining the parameters for the connected components, as we know that points in each building are spaced at $1 \mathrm{~m}$ on the horizontal plane. Further cleaning of the dataset can be done by removing isolated triangles or very small triangles that are created over sparse points. A previous cleaning step can be applied to remove outliers, i.e. isolated points. 


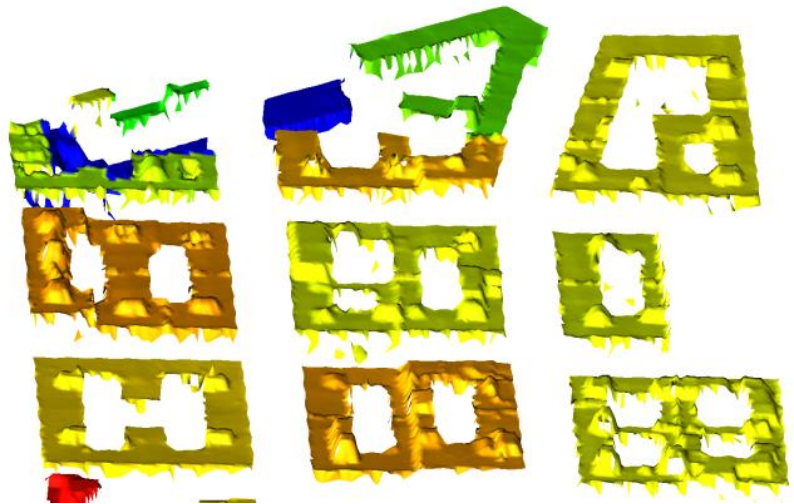

Figure 7. Triangulated surfaces over connected components.

\section{CONCLUSIONS}

The work described shows that significant information is present in commonly used LiDAR-derived products, i.e. dense DTMs and DSMs. Conversion of the unstructured point cloud to a regularly spaced grid helps in providing space-related information to support removing isolated parts that do not belong to roofs. The point sets have an id related to the building footprint thanks to spatial intersection and are thus available for further modelling of roofs to CityGML schema, and support LOD2 object creation. This last step is not the focus of this work, but several investigations are available in literature. Added value of LiDAR surveys over urban areas is a known fact, and this work further supports the idea that dense DTM/DSMs can be very important for urban city modelling.

Future work will take further steps into classification of roof types by taking descriptive features from each set of points belonging to a single building. Contextual 3D information, such as the normalized eigenvalues of the $3 \mathrm{D}$ tensor tensor matrix can be used to provide linearity, sphericity and planarity indices for each point, and then using these information for example for supervised classification of roof types. After having an idea of the roof type, primitive objects representing each type can be fitted to the point set to determine individual parameters of each roof, and then merge the roof model to the LOD1 model, creating a LOD2 object in CityGML.

\section{ACKNOWLEDGEMENTS}

This research is supported by the Italian Ministry of Education through the project of national interest (PRIN) Urban-Geo Big Data (project code: 20159CNLW8 - PE10).

\section{REFERENCES}

Agugiaro, G., 2016. Energy planning tools and CityGML-based $3 \mathrm{D}$ virtual city models: experiences from Trento (Italy). Applied Geomatics, 8, 41-56. doi:10.1007/s12518-015-0163-2

Agugiaro, G., Benner, J., Cipriano, P., Nouvel, R., 2018. The Energy Application Domain Extension for CityGML: enhancing interoperability for urban energy simulations. Open Geospatial
Data, Software and Standards,

doi:10.1186/s40965018-0042-y

Alharthy, A., Bethel, J., 2001. Heuristic Filtering and 3D Feature Extraction From Lidar Data. Civil Engineering, 6. doi:10.1.1.137.7262

Arroyo Ohori, K., Biljecki, F., Kumar, K., Ledoux, H., Stoter, J., 2018. Modeling Cities and Landscapes in 3D with CityGML, in: Building Information Modeling. Springer International Publishing, Cham, pp. 199-215. doi:10.1007/978-3319-92862-3_11

Barazzetti, L., Brovelli, M.A., Valentini, L., 2010. LiDAR digital building models for true orthophoto generation. Applied Geomatics,. doi:10.1007/s12518-010-0034-9

Biljecki, F., Kumar, K., Nagel, C., 2018. CityGML Application Domain Extension (ADE): overview of developments. Open Geospatial Data, Software and Standards, 3, 13. doi:10.1186/s40965-018-0055-6

Brovelli, A., Cina, A., Crespi, M., Lingua, A., Manzino, A., 2012. Ortoimmagini e modelli altimetrici a grande scala, in: Linee Guida, CISIS - Centro Interregionale Di Coordinamento e Documentazione per Le Informazioni Territoriali. pp. 1-129.

Brovelli, M., Boccardo, P., Crespi, M., Lanari, R., Pirotti, F., 2017. URBAN GEO-BIG DATA - URBAN GEOmatics for Bulk Information Generation, DAta assessment and Technology Awarness [WWW Document].

Dillencourt, M.B., Samet, H., Tamminen, M., 2002. A general approach to connected-component labeling for arbitrary image representations. Journal of the ACM,

doi:10.1145/128749.128750

Fissore, F., Pirotti, F., 2018. Migration of digital cartography to CityGML; a web-based tool for supporting simple ETL procedures. ISPRS - International Archives of the Photogrammetry, Remote Sensing and Spatial Information Sciences, XLII-4, 193-200. doi:10.5194/isprs-archives-XLII4-193-2018

Jayaraj, P., Ramiya, A.M., 2018. 3D CITYGML BUILDING MODELLING FROM LIDAR POINT CLOUD DATA. ISPRS International Archives of the Photogrammetry, Remote Sensing and Spatial Information Sciences, XLII-5, 175-180.

doi:10.5194/isprs-archives-XLII-5-175-2018

Labetski, A., Kumar, K., Ledoux, H., Stoter, J., 2018. A metadata ADE for CityGML. Open Geospatial Data, Software and Standards, 3, $16 . \quad$ doi:10.1186/s40965-018-0057-4

Lu, Z., Im, J., Rhee, J., Hodgson, M., 2014. Building type classification using spatial and landscape attributes derived from LiDAR remote sensing data. Landscape and Urban Planning, 130, 134-148. doi:10.1016/j.landurbplan.2014.07.005 
M., C.G., A., G., L., L., A., V., 2000. An approach to estimate of 2D-3D motion of surfaces: the frequency domain technique.

Ministero dell'Ambiente e della Tutela del Territorio e del Mare, 2019. Portale Cartografico Nazionale [WWW Document].

URL http://www.pen.minambiente.it

Morgan, M., Habib, A., 2002. Interpolation of LIDAR Data and Automatic Building Extraction 10.

Palmer, T.C., Shan, J., 2002. A Comparative Study on Urban Visualization Using LIDAR Data in GIS 14, 7.

Pirotti, F., Brovelli, M.A., Prestifilippo, G., Zamboni, G., Kilsedar, C.E., Piragnolo, M., Hogan, P., 2017. An open source virtual globe rendering engine for 3D applications: NASA World Wind. Open Geospatial Data, Software and Standards, 2, 4. doi:10.1186/s40965-017-0016-5

Pirotti, F., Ravanelli, R., Fissore, F., Masiero, A., 2018. Implementation and assessment of two density-based outlier detection methods over large spatial point clouds. Open Geospatial Data, Software and Standards, 3, 14.

doi:10.1186/s40965-018-0056-5

Pirotti, F., Zanchetta, C., Previtali, M., Della Torre, S., 2019. Detection of building roofs and facades from aerial laser scanning data using deep learning. ISPRS - International Archives of the Photogrammetry, Remote Sensing and Spatial Information Sciences, In print.

Rottensteiner, F., 2008. Automatic extraction of buildings from airborne laserscanner data and aerial images.

Rottensteiner, F., Briese, C., 2002. A new method for building extraction in urban areas from high-resolution LIDAR data. International Archives of the Photogrammetry, Remote Sensing and Spatial Information Sciences, 7.

Sengul, A., 2012. Extracting semantic building models from aerial stereo images and conversion to CityGML. ISPRS International Archives of the Photogrammetry, Remote Sensing and Spatial Information Sciences, XXXIX-B3, 321-324.

doi:10.5194/isprsarchives-XXXIX-B3-321-2012

Steed, A., Spinello, S., Croxford, B., Milton, R., 2004. Data visualization within urban models, in: Proceedings Theory and Practice of Computer Graphics, 2004. IEEE, Bournemouth, UK, pp. 9-16. doi:10.1109/TPCG.2004.1314447

TUDelft, 2019. General 3dfier tutorial to generate LOD1 models [WWW Document]. URL https://github.com/tudelft3d/3dfier

Verma, V., Kumar, R., Hsu, S., 2006. 3D Building Detection and Modeling from Aerial LIDAR Data, in: Proceedings of the 2006 IEEE Computer Society Conference on Computer Vision and Pattern Recognition - Volume 2, CVPR '06. IEEE Computer Society, Washington, DC, USA, pp. 2213-2220.

doi:10.1109/CVPR.2006.12
Vosselman, G., 2013. Point cloud segmentation for urban scene classification, in: International Archives of the Photogrammetry, Remote Sensing and Spatial Information Sciences - ISPRS Archives. doi:10.5194/isprsarchives-XL-7-W2-257-2013

Weinmann, M., Jutzi, B., Hinz, S., Mallet, C., 2015. Semantic point cloud interpretation based on optimal neighborhoods, relevant features and efficient classifiers. ISPRS Journal of Photogrammetry and Remote Sensing, 105, 286-304. doi:10.1016/j.isprsjprs.2015.01.016

Zeng, Q., 2008. Data Filtering and Feature Extraction of Urban Typical Objects From Airborne Lidar Point Cloud. The International Archives of the Photogrammetry, Remote Sensing and Spatial Information Sciences,. 